\title{
Signalkompensation mittels Gate-Potential bei gassensitiven Feldeffekttransistoren
}

\author{
M. Bastuck ${ }^{1,2}$, C. Daut ${ }^{1}$, A. Schütze ${ }^{1}$ \\ ${ }^{1}$ Lehrstuhl für Messtechnik, Universität des Saarlandes, Saarbrücken, Deutschland \\ ${ }^{2}$ Applied Sensor Science, Sensor and Actuator Systems, Linköping University, Linköping, Schweden \\ m.bastuck@Imt.uni-saarland.de
}

\begin{abstract}
Zusammenfassung
In dieser Arbeit wird ein Kompensationsbetrieb für gassensitive Feldeffekttransistoren implementiert, indem der Strom durch den Sensor mittels Regelung des Gate-Potentials konstant gehalten wird. Die Änderung im Gate-Potential spiegelt dabei direkt die Änderung des Potentials der Sensoroberfläche durch das Gas wider. Im Vergleich mit dem nicht kompensierten Betriebsmodus, d. h. direkte Messung des Stroms ohne Beeinflussung des Gate-Potentials, weist der kompensierte Modus eine bessere relative Sensorantwort bei größeren Konzentrationen und ein deutlich lineareres Verhalten sowohl im statischen als auch im temperaturzyklischen Betrieb auf. Als Erklärung für diese Unterschiede schlagen wir den Transport von Sauerstoffanionen, beeinflusst durch sich ändernde elektrische Felder, auf der Oberfläche vor.
\end{abstract}

Keywords: GasFET, SiC-FET, Signalkompensation, TCO, lineare Regression

\section{Einführung}

Temperaturzyklischer Betrieb (temperature cycled operation, TCO) wird häufig eingesetzt, um die Sensitivität und Selektivität chemischer Sensoren, insbesondere Gassensoren, zu steigern [1,2]. Oft werden hierfür HalbleiterGassensoren (metal-oxide semiconductor, MOS) verwendet: sie sind aufgrund ihres einfachen Aufbaus relativ leicht herzustellen und auszulesen. Im Gegensatz dazu steht der deutlich komplexere Aufbau von gassensitiven Feldeffekttransistoren (GasFETs), z. B. auf Basis von Siliziumcarbid ( $\mathrm{SiC}$ ). Dieses Material eignet sich durch die große Bandlücke für Betrieb bei erhöhter Temperatur und ist zudem chemisch inert, so dass ein Betrieb direkt in Abgas möglich ist. Relevante Zielgase sind daher Kohlenmonoxid (CO), Stickoxide ( $\mathrm{NO}_{\mathrm{x}}$ ) und Kohlenwasserstoffe (HC), aber auch Ammoniak $\left(\mathrm{NH}_{3}\right)$ für die Überwachung von SCR-Abgasreinigungssystemen [3]. Relevante Konzentrationen liegen im Bereich von 1520 ppm für Ammoniak.

GasFETs bieten ergänzend zu den Parametern von MOS-Sensoren (Strom, Spannung, Temperatur) zusätzliche Freiheitsgrade in Form des Gate- und Substrat-Potentials [3]. Interessant ist insbesondere das Gate-Potential, da es direkt am Katalysator anliegt und so chemische Reaktionen und Ladungstransport auf der Sensoroberfläche direkt beeinflussen kann. Wie beim temperaturzyklischen Betrieb können so die Eigenschaften des Sensors im Betrieb aktiv manipuliert und damit ein multidimensionales Signal mit zusätzlichen Informationen generiert werden.

In dieser Arbeit wurde ein Regler implementiert, der das eigentliche Sensorsignal, d. h. den Drain-Strom ID bei einer vorgegebenen Drain-Source-Spannung UDS, durch Regelung des Gate-Potentials UGS konstant hält, d. h. das Signal kompensiert. Die Änderung im Gate-Potential entspricht somit direkt der durch das Gas verursachten Potentialänderung der Sensoroberfläche. Die Charakterisierung dieses neuen, kompensierten Betriebsmodus zeigt eine verbesserte relative Sensorantwort bei höheren Konzentrationen sowie einen deutlich lineareren Zusammenhang zwischen Gaskonzentration und Sensorantwort verglichen mit dem nicht kompensierten Modus.

\section{Sensor}

Als Sensor wurde ein gassensitiver SiCFeldeffekttransistor verwendet [3,4]. Sein Aufbau ist prinzipiell identisch zu einem herkömmlichen, selbstleitenden n-Kanal-FET mit dem Unterschied, dass auf dem Gateoxid 
(Siliziumdioxid, $\mathrm{SiO}_{2}$ ) ein poröser Katalysator, hier Platin, aufgebracht ist (Abb. 1). Die Porosität wird durch kurzes DCMagnetronsputtern (ca. $60 \mathrm{~s}$ ) bei erhöhtem Druck (Argon, ca. 6·10-3 mbar) erreicht.

Das Funktionsprinzip basiert auf der Dissoziation von Gasmolekülen am Katalysator bzw. an den Dreiphasengrenzen Katalysator/Oxid/Gas. Dabei abgespaltene Protonen $\left(\mathrm{H}^{+}\right)$diffundieren zur Grenzfläche zwischen Katalysator und Oxid bzw. durch spill-over direkt auf das Oxid und bilden dort polare $\mathrm{OH}-G r u p p e n$, wodurch ein elektrisches Feld entsteht, das die Leitfähigkeit zwischen Drain und Source beeinflusst (hier: erhöht). Nicht wasserstoffhaltige Gase können ebenfalls detektiert werden, indem sie die Bedeckung des Katalysators mit negativ geladenen Sauerstoffionen erhöhen (oxidierende Gase) oder verringern (reduzierende Gase). Diese Bedeckung überträgt sich durch spill-over auf das Oxid, wo die negativ geladenen lonen die Leitfähigkeit der Drain-Source-Strecke verringern. Weiterhin können Gasmoleküle auch direkt am Oxid adsorbieren und das elektrische Feld eines Sauerstoffions bzw. einer $\mathrm{OH}-G r u p p e$ durch ihr eigenes Dipolfeld ersetzen [5].

Im Allgemeinen erfolgt die Besetzung der Oberfläche entlang einer Temkin-Adsorptionsisotherme [4] und folgt damit einem logarithmischen Verlauf, der sich auch in der Abhängigkeit der Sensorantwort von der Gaskonzentration widerspiegelt.

Zur Beschleunigung bzw. Aktivierung der chemischen Reaktionen auf der Sensoroberfläche wird der Sensorchip durch einen Keramikheizer auf Temperaturen von, je nach Anwendungsfall, zwischen 100 und $400{ }^{\circ} \mathrm{C}$ gebracht.

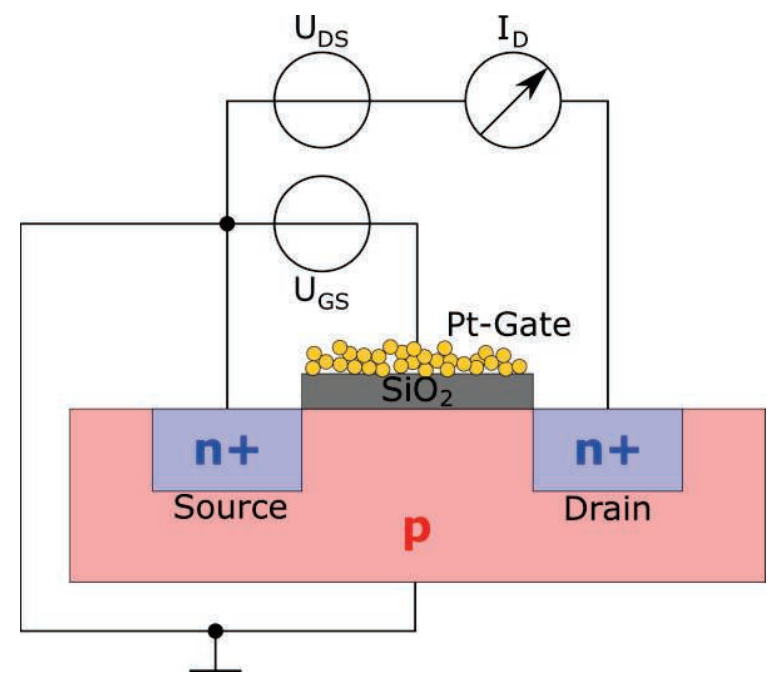

Abb. 1. FET-Struktur mit elektrischer Verschaltung und porösem Platin-Gate.

\section{Experimenteller Aufbau}

Zur Charakterisierung des Sensors wurde eine Gasmischanlage (GMA) ähnlich zu der in [6] beschriebenen genutzt. Dabei wird einem Trägergas, hier Luft aus einem Nullluftgenerator, eine geringe Konzentration des Testgases, hier Ammoniak aus einer Gasflasche mit 3000 ppm in synthetischer Luft, zugemischt. Die Zumischung erfolgt computergesteuert und wird so geregelt, dass der Gesamtfluss über den Sensor immer bei $500 \mathrm{ml} / \mathrm{min}$ bleibt. Auf diese Weise wurden am Sensor Konzentrationen von 6, 12, 18, 24 und $30 \mathrm{ppm}$ Ammoniak in trockener Luft erzeugt. Jede Konzentration wurde, zeitlich versetzt, zweimal angeboten, sodass der Einfluss von Sensordrift in statistischen Modellen reduziert werden kann [7].

Am Sensor wurde eine Spannung $U_{D S}=4 \mathrm{~V}$ zwischen Drain und Source angelegt und der fließende Strom ID gemessen. Die Messung wurde zweimal durchgeführt, je einmal mit einem der beiden unterschiedlichen Betriebsmodi: im nicht kompensierten Modus wird der gemessene Strom direkt als Sensorsignal ausgewertet. Im kompensierten Modus wird das Potential des Gates mittels PIRegler so eingestellt, dass der Strom ID auf einem konstanten Wert gehalten wird. Alle Parameter des Sensors können über eine speziell entwickelte Hardwareplattform (3S $\mathrm{GmbH}$, Saarbrücken) eingestellt und ausgelesen werden. Die Hardwareplattform kommuniziert mit einem eigens geschriebenen LabVIEW-Programm. Für die hier vorgestellte Charakterisierung des kompensierten Modus wurden $U_{D S}$ und ID sowie $U_{G S}$ mit einem SourceMeter 2602B (Keithley Instruments) eingestellt bzw. gemessen. Das SourceMeter bietet eine um drei Größenordnungen bessere Auflösung $(50 \mu \mathrm{V})$ beim Einstellen des GatePotentials als die Hardwareplattform, die nicht für diesen Betriebsmodus ausgelegt wurde. Die Vorgabe der Temperatur erfolgt in allen Fällen durch die Hardwareplattform.

Während der Messung wurde die Temperatur des Sensors innerhalb von $25 \mathrm{~s}$ von 180 auf $270{ }^{\circ} \mathrm{C}$ erhöht und anschließend innerhalb von $35 \mathrm{~s}$ wieder auf $180^{\circ} \mathrm{C}$ abgesenkt (Abb: 2), sodass ein Zyklus eine Minute bzw., bei einer Abtastfrequenz von $10 \mathrm{~Hz}, 600$ Datenpunkte lang ist. Dieser Zyklus wurde während der gesamten Messung wiederholt.

\section{Datenauswertung}

In der Auswertung wurden aus dem zyklischen Sensorsignal Merkmale berechnet, die die Form des Signals mit möglichst wenigen Parametern möglichst gut beschreiben. In diesem Fall wurden der Mittelwert des 
kompletten Zyklus sowie die Steigung in vier Bereichen (Abb. 2, graue Bereiche) berechnet. Durch dieses Vorgehen werden die Unterschiede in der Auflösung der Strommessung zwischen nicht kompensiertem (Hardwareplattform, $60 \mathrm{nA}$ ) und kompensiertem (SourceMeter, $20 \mathrm{nA}$ ) Modus bereits weitestgehend eliminiert. Zusätzlich wurde, für einen fairen Vergleich der beiden Modi, ein normalverteiltes Rauschen zu den Rohdaten des SourceMeters addiert, um einen möglichen Einfluss der etwas besseren Auflösung auf das Ergebnis auszugleichen.

Mittels LDA (Lineare Diskriminanzanalyse) wird die Dimension des so entstandenen Merkmalsraums von fünf auf eins reduziert. Die LDA findet dabei die Projektion, in der gleiche Konzentrationen möglichst nah zusammen, und ungleiche Konzentrationen möglichst weit voneinander entfernt liegen [8].

Aus den zyklischen Daten kann andererseits ein quasistatisches Signal generiert werden, welches dem Sensorsignal bei einer festen Temperatur entspricht, indem aus jedem Zyklus der Messpunkt bei der gewünschten Temperatur aufgetragen wird. In diesem quasistatischen Signal wurde die Höhe der Sensorantwort abhängig von der angebotenen Ammoniakkonzentration bestimmt, indem der Mittelwert der letzten Messpunkte unmittelbar vor Ende des Gasangebots vom Mittelwert der letzten Messpunkte unmittelbar vor Beginn des Gasangebots subtrahiert wurde. Das Sensorsignal ist dabei im nicht kompensierten Modus der Strom ID und im kompensierten Modus das Gate-Potential UGs.

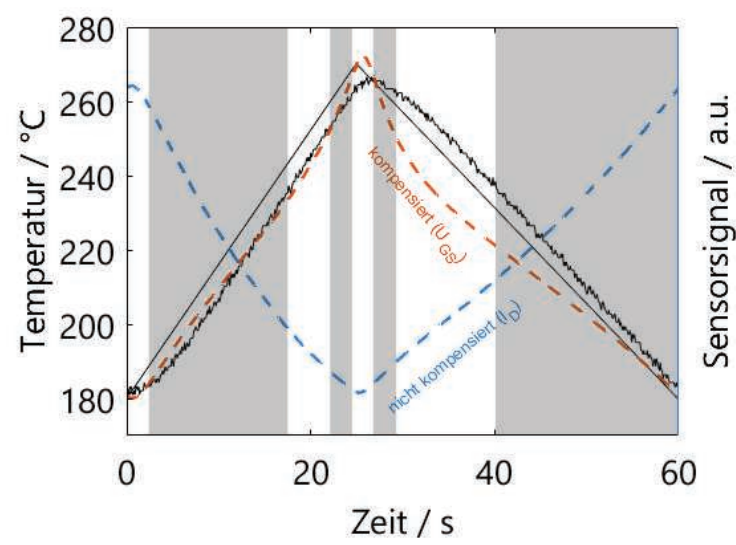

Abb. 2. Temperaturzyklus mit vorgegebener und rückgemessener Temperatur (schwarz, durchgehend) und die resultierende Form des Sensorsignals in beiden Betriebsmodi (gestrichelt). Als Merkmale wurden die Steigungen in den grauen Bereichen sowie der Mittelwert des gesamten Zyklus berechnet.
An die so erhaltenen Werte wird jeweils ein lineares und ein logarithmisches Modell angepasst und das Bestimmtheitsmaß $\mathrm{R}^{2}$ berechnet. Ein Wert nahe 1 bedeutet eine gute Passung des Modells an die Daten, ein Wert nahe 0 keine Korrelation zwischen Daten und Modell.

\section{Ergebnisse und Diskussion}

Um die Performance des PI-Reglers zu testen, wurde zunächst eine Änderung des Sensorstroms erzeugt, indem die Temperatur des Sensors verändert wurde ohne Veränderung der Gasbedeckung. Zwischen Temperatur und Strom besteht eine negative Korrelation, d. h. eine niedrigere Temperatur führt zu einem höheren Strom bei ansonsten gleichen Bedingungen. Die Aufgabe des PIReglers ist es, das Gate-Potential so anzupassen, dass der Strom trotz geänderter Temperatur konstant bleibt. Strom und GatePotential korrelieren positiv, woraus folgt, dass das Gate-Potential mit steigender Temperatur erhöht werden muss, um den Strom konstant zu halten. In Abb. 3 wurde die Temperatur in Schritten von $10^{\circ} \mathrm{C}$ zwischen 240 und $200{ }^{\circ} \mathrm{C}$ variiert, während der Strom ID mit Zielwert $380 \mu \mathrm{A}$ und das resultierende Gate-Potential UGs aufgetragen wurden.

Aufgrund der relativ großen thermischen Masse des Aufbaus folgt die tatsächliche der vorgegebenen Temperatur mit einer Zeitkonstante von etwa t90 $=5 \mathrm{~s}$. Der Ausgleich der sich nach dem Temperatursprung ergebenen Regelabweichung ist, unabhängig von der absoluten Temperatur, ebenfalls nach etwa $5 \mathrm{~s}$ abgeschlossen und hat damit keinen nennenswerten, negativen Einfluss auf die Reaktionszeit des Sensors auf Gasangebote, die im Bereich von Minuten liegt. Die Höhe der Regelabweichung korreliert mit der absoluten Temperatur, was damit erklärt werden kann, dass die Sensoroberfläche bei hohen Temperaturen schneller abkühlt und damit die Signaländerung größer wird. Dieses schnellere Abkühlen zeigt sich nicht in der gemessenen Temperatur, da der derzeit eingesetzte Pt-100 lediglich die Temperatur des Keramikheizers misst. Derzeit ist unklar, ob die Drift, die sich im Sensorsignal ( $U_{G S}$ ) insbesondere für hohe Temperaturen zeigt, durch lonentransport auf der Sensoroberfläche oder durch langsame, elektronische Ausgleichsprozesse innerhalb der FET-Struktur zu erklären ist.

Insgesamt zeigt sich eine zufriedenstellende Performance des Reglers. Für einen temperaturzyklischen Betrieb sollte die kurze Einschwingzeit des Signals nach Sprüngen beachtet werden. Alternativ können 


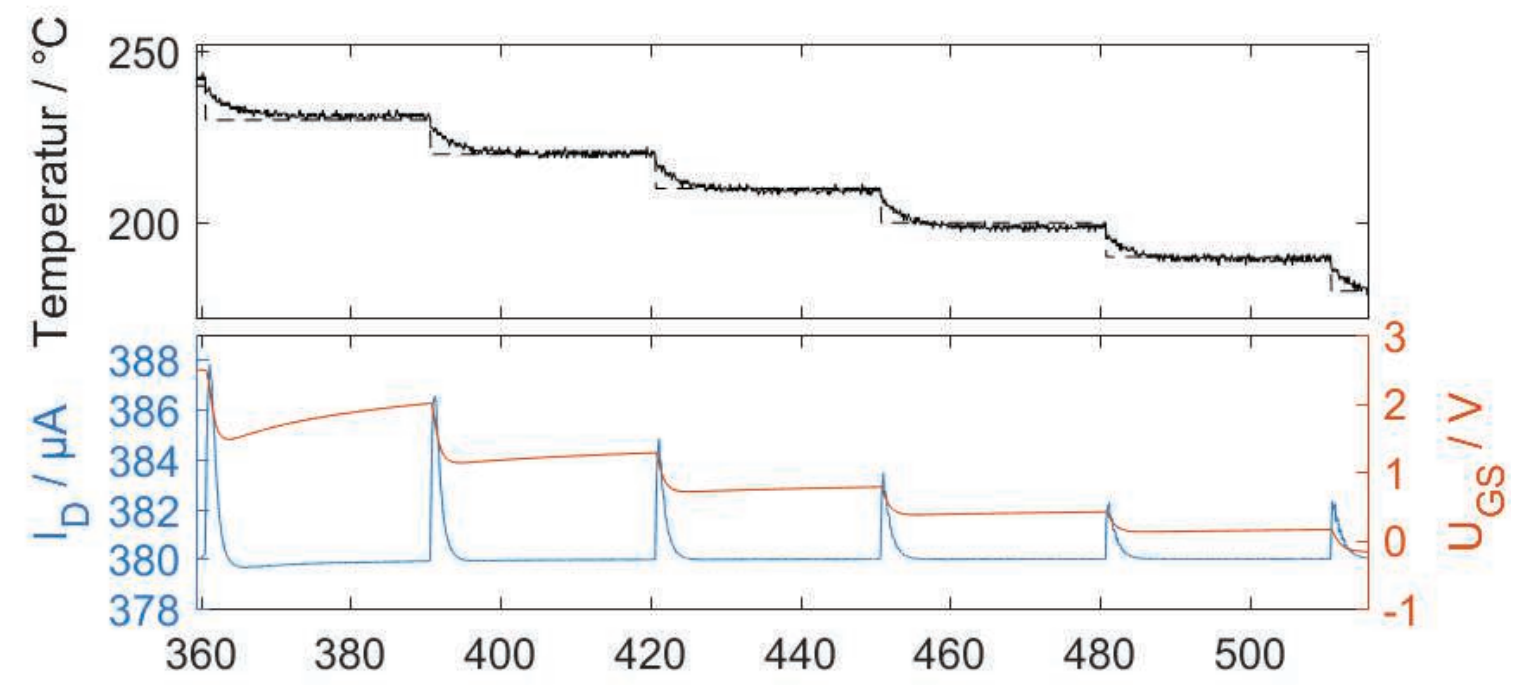

Abb. 3. Heizen des Sensors auf verschiedene Temperaturen, während der PI-Regler den DrainStrom I durch Anpassen der Gate-Spannung $U_{G S}$ konstant hält. Die Signaländerung durch ein Gasangebot entspricht qualitativ der Signaländerung durch Änderung der Sensortemperatur. Bei höheren Temperaturen und höheren $U_{G S}$ zeigt sich eine deutliche Zeitabhängigkeit von $U_{G S}$, die durch lonentransport auf der Oberfläche erklärt wird, vermutlich zwischen Isolator und Katalysatoroberfläche.

rampenförmige Zyklen wie in Abb. 2 verwendet werden, um Sprünge gänzlich zu vermeiden.

Zum Vergleich zwischen kompensiertem und nicht kompensiertem Betriebsmodus wurde der Sensor sechs verschiedenen Ammoniakkonzentrationen zwischen 0 und $30 \mathrm{ppm}$ ausgesetzt. In beiden Fällen wurde ein Temperaturzyklus wie in Abb. 2 dargestellt verwendet.

Im Histogramm der ersten Diskriminanzfunktion (Abb. 4) zeigt sich, dass reine Luft (violett, $0 \mathrm{ppm}$ Ammoniak) im nicht kompensierten Modus deutlich weiter von allen Gasangeboten mit Ammoniak (blau) abgesetzt ist, während die Abstände zwischen den einzelnen Gasangeboten nur geringfügig variieren. Dies lässt die Vermutung zu, dass der kompensierte dem nicht kompensierten Modus für noch kleinere Gaskonzentrationen unterlegen ist.

Eine mögliche Erklärung für den geringeren Abstand zwischen Luft- und Gasgruppen ist eine Signalkompensation durch die Verschiebung von negativ geladenen Sauerstoffionen auf der Oberfläche. Im thermodynamischen Gleichgewicht und für $U_{G S}=0 \mathrm{~V}$ ist das Verhältnis zwischen Bedeckung von Katalysator und Isolator mit Sauerstoffionen konstant. Werden durch Ammoniak Protonen eingebracht, erhöht sich der Strom durch den Sensor, worauf der PIRegler mit einer Verringerung des GatePotentials reagiert. Dadurch verschiebt sich das Verhältnis der Bedeckungen in Richtung des Isolators, da die negativen Sauerstoffionen von dem negativer gewordenen Katalysator auf den Isolator abwandern. Der Einfluss der zusätzlichen negativen Ladungen kompensiert den Einfluss der Protonen teilweise, sodass das Gate-Potential, d. h. das Sensorsignal, weniger stark abgeregelt werden muss.

Ein direkter Vorteil des kompensierten Modus ist seine verbesserte Linearität. Diese deutet sich in den Histogrammen in Abb. 4 bereits durch die nahezu äquidistanten Abstände der Gruppen zueinander an. Zur Bestätigung wurde die Sensorantwort in Abhängigkeit der Konzentration im quasistatischen Signal bei $270{ }^{\circ} \mathrm{C}$ bestimmt (Abb. 5). Im nicht kompensierten Modus erklärt ein logarithmisches Modell die Daten deutlich besser als ein lineares, was aufgrund der Temkin-Adsorptionsisotherme zu erwarten ist. Im kompensierten Modus konnten hingegen keine sinnvollen Parameter für ein logarithmisches Modell gefunden werden, und ein lineares Modell erklärt bereits denselben Anteil an Variation in den Daten wie das logarithmische Modell zuvor. Gleichzeitig weist der kompensierte Modus mit $8 \%$ bei 30 ppm eine etwas höhere Sensorantwort als der nicht kompensierte Modus ( $6 \%$ bei $30 \mathrm{ppm}$ ) auf, was einer linearen Fortsetzung der Anfangssteigung im nicht kompensierten Modus entspricht. 


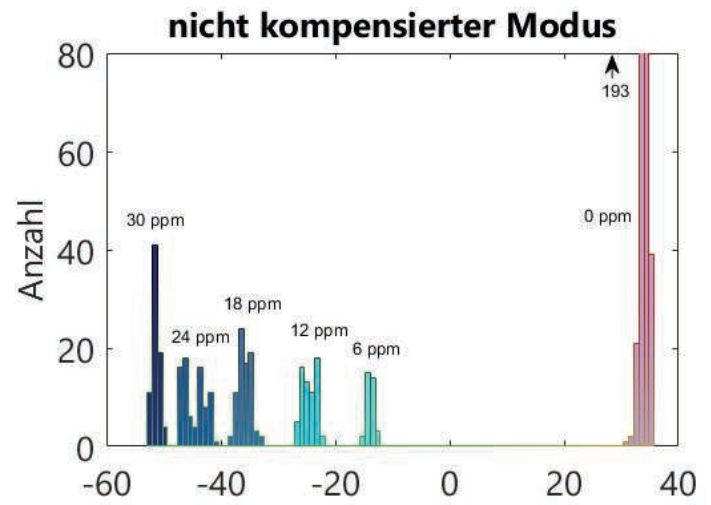

1. Diskriminanzfunktion

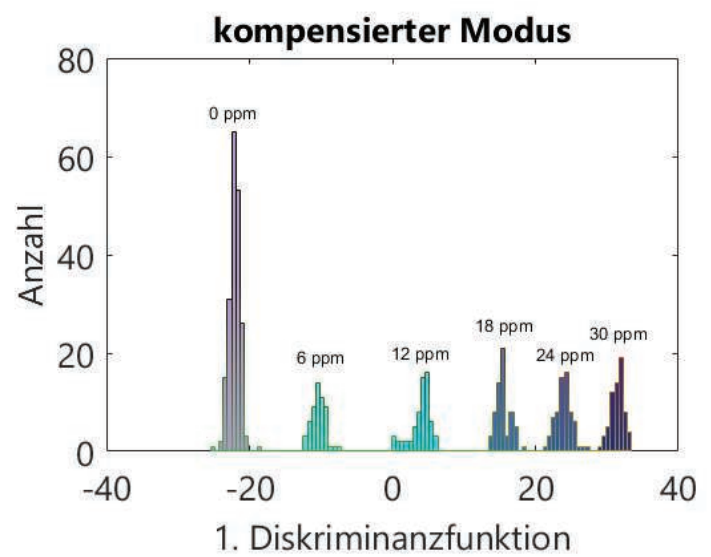

Abb. 4. Erste Diskriminanzfunktion einer LDA mit den fünf berechneten Merkmalen für beide Betriebsmodi. Auffällig ist der große Abstand zwischen Luft und Gasangeboten für den nicht kompensierten Modus.

Ein lineares Verhalten ist wünschenswert, da es zahlreiche Vorteile mit sich bringt, wie unter anderem die deutlich einfachere mathematische und statistische Handhabung sowie eine konzentrationsunabhängige Empfindlichkeitsangabe des Sensors.

\section{Fazit und Ausblick}

Es wurde ein neuer Betriebsmodus für gassensitive Feldeffekttransistoren implementiert und charakterisiert, bei dem der sonst als Sensorsignal genutzte Strom durch eine Nachregelung des Gate-Potentials konstant gehalten wird. Die Zeitkonstante dieser Regelung liegt im Bereich weniger Sekunden, während die Zeit bis zum Endwert der Gasantwort mehrere Minuten betragen kann, so dass die Regelung keinen nennenswerten Einfluss auf die Zeitkonstante des Sensors hat. In diesem kompensierten Betriebsmodus beeinflussen kleine Gaskonzentrationen das Sensorsignal weniger stark als im nicht kompensierten Modus, wodurch sich letztendlich ein deutlich linearerer Zusammenhang
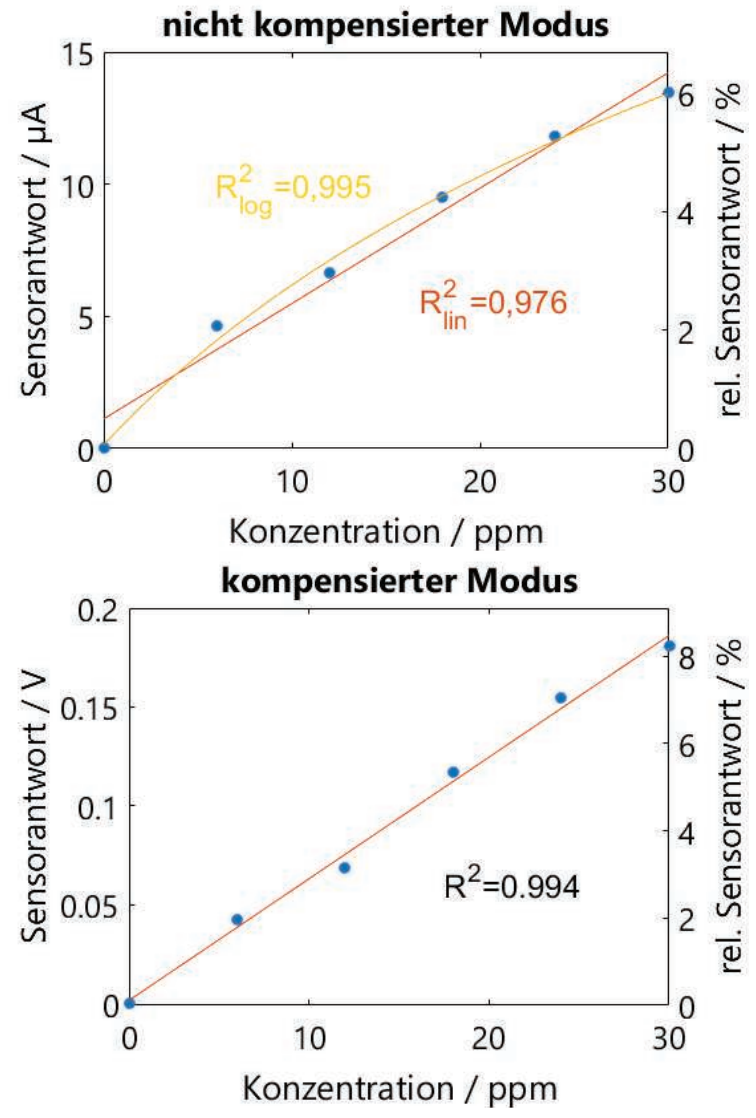

Abb. 5. Abhängigkeit des Sensorsignals von der Ammoniakkonzentration für beide Betriebsmodi. Die Datenpunkte können im kompensierten Modus deutlich besser durch ein lineares Modell erklärt werden.

zwischen Gaskonzentration und Sensorantwort ergibt. Als Erklärung hierfür schlagen wir einen elektrostatischen Einfluss auf die auf der Oberfläche stattfindenden (reverse) spill-overEffekte von Sauerstoffanionen vor. Die zusätzlichen negativen Ladungen auf dem Isolator könnten den Einfluss des Gases teilweise kompensieren.

Eine deutlich geringere Distanz zwischen Luftund Gasgruppen konnte, als Indikator für verbesserte Linearität, bereits in weiteren Messungen beobachtet werden. Die tatsächliche Linearität bzw. der lineare Bereich soll in Zukunft mit weiteren Messungen über einen deutlich größeren Konzentrationsbereich und für weitere Gase untersucht werden.

\section{Danksagungen}

Die Autoren danken der Firma SenSiC AB (Kista, Schweden) für die Bereitstellung der SiC-FET-Sensoren sowie der Firma $3 \mathrm{~S} \mathrm{GmbH}$, insbesondere Wolfhard Reimringer, für die Entwicklung und Betreuung der Hardwareplattformen. 


\section{Literaturnachweis}

[1] P. Reimann, A. Schütze, Sensor Arrays, Virtual Multisensors, Data Fusion, and Gas Sensor Data Evaluation, in: C.-D. Kohl, T. Wagner (Eds.), Gas Sens. Fundam., Springer Berlin Heidelberg, Berlin, Heidelberg, 2014: pp. 67107. doi:10.1007/978-3-642-54519-1.

[2] T. Baur, A. Schütze, T. Sauerwald, Optimierung des temperaturzyklischen Betriebs von Halbleitergassensoren, Tm - Tech. Mess. 82 (2015) 187-195. doi:10.1515/teme-2014-0007.

[3] M. Andersson, R. Pearce, A.L. Spetz, New generation $\mathrm{SiC}$ based field effect transistor gas sensors, Sensors Actuators, B Chem. 179 (2013) 95-106. doi:10.1016/j.snb.2012.12.059.

[4] I. Lundström, H. Sundgren, F. Winquist, M. Eriksson, C. Krantz-Rülcker, A.L. Spetz, Twenty-five years of field effect gas sensor research in Linköping, Sensors Actuators, B Chem. 121 (2007) 247-262. doi:10.1016/j.snb.2006.09.046.

[5] J. Schalwig, P. Kreisl, S. Ahlers, G. Müller, Response mechanism of SiC-based MOS fieldeffect gas sensors, IEEE Sens. J. 2 (2002) 394-402. doi:10.1109/JSEN.2002.806214.

[6] N. Helwig, M. Schüler, C. Bur, A. Schütze, T. Sauerwald, Gas mixing apparatus for automated gas sensor characterization, Meas. Sci. Technol. 25 (2014) 55903. doi:10.1088/0957-0233/25/5/055903.

[7] C. Bur, M. Bastuck, A.L. Spetz, M. Andersson, A. Schütze, Selectivity enhancement of SiCFET gas sensors by combiningtemperature and gate bias cycled operation using multivariate statistics, Sensors Actuators, B Chem. 193 (2014) 931-940. doi:10.1016/j.snb.2013.12.030.

[8] R. Gutierrez-Osuna, Pattern analysis for machine olfaction: A review, IEEE Sens. J. 2 (2002) 189-202.

doi:10.1109/JSEN.2002.800688. 